\title{
Der nahe und der ferne Euler
}

\author{
Norbert Schappacher
}

\section{Einleitung}

Das gleichmässige Metronom des Kalenders, das am 15. April dieses Jahres 2007 auf den 300. Geburtstag Leonhard Eulers anschlägt, lässt uns dieses Gelehrten aus Basel gedenken, des grössten Mathematikers und wahrscheinlich produktivsten Naturwissenschaftlers des 18. Jahrhunderts. Die ersten beiden grossen Élogen auf Euler: die lange, teils persönliche Petersburger Rede des Schweizer Landsmanns und Sekretärs der letzten zehn Jahre Eulers, Nicolas Fuss vom 23.10.1783, gut einen Monat nach Eulers Tod (18.9.1783), und die kongenialere Schilderung vor der Pariser Akademie durch Marie Jean Antoine Nicolas Caritat, Marquis de Condorcet, am 6.2.1785, wandten sich an ein Publikum, das noch unter dem unmittelbaren Einfluss von Eulers Lehrbüchern und Arbeiten stand. Gut 120 Jahre später, bei der zweihundertsten Wiederkehr von Eulers Geburtstag 1907, hatte sich zwischen Euler und die, die ihn gerne würdigten, schon die kolossale Entwicklung der Mathematik im 19. Jahrhundert geschoben. So enthält die Festschrift zur Feier des 200. Geburtstages Leonhard Eulers ${ }^{1}$ unter anderem ${ }^{2}$ einen gedrängten Überblick aus der Feder Felix Müllers Über bahnbrechende Arbeiten Leonhard Eulers aus der reinen Mathematik, der sich vor allem bemüht, Euler als Vorläufer von Forschungen zu beschreiben, die 1907 aktuell waren. $\mathrm{Zu}$ den zweibändigen Institutiones calculi differentialis cum eius usu in analysi infinitorum ac doctrina serierum ${ }^{3}$, dem zweiten Teil von Eulers einflussreicher

\footnotetext{
${ }^{1}$ Erschienen 1907 im Teubner Verlag (Leipzig \& Berlin) als XXV. Heft der von Moritz Cantor begründeten Abhandlungen zur Geschichte der mathematischen Wissenschaften mit Einschluss ihrer Anwendungen. Der Band beruht zum Teil auf der Festsitzung, die die Berliner Mathematische Gesellschaft genau am 15.4.1907 im Grossen Auditorium des Physikalischen Instituts der Berliner Universität abgehalten hatte.

${ }^{2}$ Nämlich nach einem biographischen Beitrag von G. Valentin über Leonhard Euler in Berlin, sowie nach dem anfangs ins 17. Jahrhundert zurückgreifenden und dann bald zur philosophischen Auseinandersetzung um Maupertuis' Prinzip der kleinsten Aktion kommenden Aufsatz A. Knesers über Euler und die Variationsrechnung - später kam Adolf Kneser besonders auf die philosophische Seite dieses Themas in seinem Buch [20] ausführlicher zurück - und bevor E. Lampe im letzten Kapitel der Entstehung der Begriffe der Exponentialfunktion und der logarithmischen Funktion eines komplexen Arguments besonders anhand von Eulers Korrespondenz mit d'Alembert nachgeht.

${ }^{3}$ E212 (St. Petersburg 1755; OO I.10). - Wir zitieren Eulers Arbeiten in erster Linie nach ihrer Nummer in Eneströms Verzeichnis, geben aber dazu in Klammern das Erscheinungsjahr (bei Büchern auch den
} 
Lehrbuchtrilogie zur Analysis ${ }^{4}$ schreibt Müller, in Anspielung auf Eulers eigene Bemerkung, das Werk halte sich ,durchaus innerhalb der Grenzen der reinen Analyse“5:

Dadurch bereitet Euler den Boden, auf dem eine Arithmetisierung der Analysis, die mit Lagrange begonnen, möglich war. Durch Einführung des neueren Grenzbegriffes entwickelte Cauchy die Arithmetisierung weiter, die dann Weierstraß vollendete.

Seit etwa 50 Jahren wenden sich Mathematikhistoriker aus gutem Grund gegen solche grosszügige Rückprojizierung der Arithmetisierung bis in das 18. Jahrhundert und konstatieren vielmehr einen doppelten Bruch in der Geschichte der Analysis ${ }^{6}$ : Einerseits löste der junge Euler die Analysis bewusst aus der Verquickung mit der Geometrie, indem er nicht mehr Kurven als Lösungen analytischer Probleme anerkannte, sondern einzig algebraischanalytische Ausdrücke, die als natürlich gegeben genommen und manipuliert wurden. Andererseits wurde diese so von Euler und Lagrange praktizierte Algebraische Analysis dann im 19. Jahrhundert durch Cauchys Aufbau abgelöst, für den Konvergenz und Grenzwerte zentral sind und in dessen Folge Existenzaussagen wie der Mittelwertsatz - welche bei Euler gar keine Rolle spielen - zu Schaltstellen der Beweise avancieren.

Diese Einsichten der Mathematikhistoriker schenken Euler einerseits seinem Jahrhundert zurück und erlauben andererseits eine unverkrampftere Lektüre seiner analytischen Traktate, die sich auf den Kalkül einlasssen kann, ohne jederzeit sklavisch Weierstraß'sche $\epsilon \mathrm{s}$ und $\delta$ s zwischen die Zeilen zu schieben. In einem charmanten kleinen Aufsatz zu Eulers Begriff der Ableitung ${ }^{7}$ hat H.M. Edwards aus Anlass dieses Eulerjahres vorgeführt, wie man durch eine solche Lesart mitunter eine grosse Nähe zu Eulers Texten gewinnen kann. Dies, obwohl das Ziel der Historiker zunächst nur darin bestand, Euler an seinen geschichtlichen Platz zurückzustellen.

Überhaupt stellt sich manchmal das Gefühl der Nähe zum Eulerschen Text durch eine glückliche Fügung ein. So konnte Günter Ziegler bei seinem Vortrag am International Euler Symposium in Basel (31.5-1.6.2007) mühelos eine Brücke von Eulers erster Erwähnung der berühmten Polyeder-Formel ${ }^{8}$ zu den heutigen Eingrenzungen verschiedener Typen von Polytopen durch Ungleichungen schlagen. Denn in jenem Brief an Gold-

Ort, bei Aufsätzen auch das Jahr der Entstehung) sowie Reihe und Band der Opera Omnia (,OO“) an, wo sie abgedruckt sind. Angaben zu den Eneström Nummern erhält man auch elektronisch über http:// www.math.dartmouth.edu/ euler/

${ }^{4}$ Nach der zweibändigen Introductio in analysin infinitorum, E101, 102 (Lausanne 1748; OO I.8, I.9), und vor den dreibändigen Institutiones calculi integralis, E342, E366, E385 (St. Petersburg 1768, 1769, 1770 ; OO I.11, I.12, I.13).

${ }^{5}$ Ich verbessere den offenkundigen Druckfehler in Müllers Text, S. 86, in dem es ,Arithmetisierung der Arithmetik“ heisst. Die Arithmetisierung der Analysis gehört ins letzte Drittel des 19. Jahrhunderts; siehe etwa [23]. Ihr Anfang wurde auch von anderen Autoren zurückverlängert; siehe etwa [4].

${ }^{6}$ Siehe beispielhaft [9]. Vgl. überblicksartig [13], sowie das Kapitel 4, „Die Algebraische Analysis des 18. Jahrhunderts" in [19, S. 131-170].

${ }^{7}$ Siehe [8].

${ }^{8}$ Siehe Eulers Brief an Goldbach vom 14.11.1750, [10, S. 332-333], wo Euler dieses Thema mit Worten einführt, deren scheinbare Beiläufigkeit sich oft findet, wenn er einen neuen Gegenstand anpackt: „Neulich kam mir in den Sinn, die allgemeinsten Eigenschaften der Körper, welche hedris planis eingeschlossen sind, zu bestimmen,..." 
bach schrieb Euler nicht nur seine mittlerweile schon auf zwei Briefmarken reproduzierte ${ }^{9}$ Formel an, und zwar in der Form $H+S=A+2$, wobei $H$ die Anzahl der Seiten - hedrae - des (konvexen) Polyeders bezeichnet, $S$ die Anzahl der Ecken - die Euler als anguli solidi sieht - und $A$ die Anzahl der Kanten, die er ,aus Mangel eines recipierten Worts, acies " nannte. Sondern er notierte gleich im selben Zug, dass auch die vier Ungleichungen gelten:

$$
A+6 \leq 3 H \quad \Leftrightarrow \quad S+4 \leq 2 H \quad \text { und } \quad A+6 \leq 3 S \quad \Leftrightarrow \quad H+4 \leq 2 S \text {. }
$$

Aber Gefühle der Nähe und Vertrautheit können auch schmerzlich trügen. Kenntnisse über das 18. Jahrhundert sind bei der Annäherung an Euler ebenso nötig wie aufgeweckte Skepsis gegenüber den eigenen Lesegewohnheiten. Insbesondere reicht es nicht, Formeln bei Euler wiederzuerkennen, mit denen wir viel verbinden.

Blättert man zum Beispiel im Aufsatz des jungen Euler: De Progressionibus transcendentibus seu quarum termini generales algebraice dari nequeunt ${ }^{10}$ bis zum $\S 14$, so findet sich für den ,allgemeinen Term der Progression 1, 2, 6, 24, 120 etc.“ die Formel: $\int d x(-l x)^{n}$. Die können wir wiedererkennen - zumal Euler in dieser Arbeit durchaus nichtganze $n$, insbesondere $n=\frac{1}{2}$, betrachtet - , weil wir entweder auswendig wissen, dass

$$
\int_{0}^{1}(-\log x)^{s} d x=\Gamma(s+1) \quad(s>-1)
$$

ist $^{11}$, oder indem wir dieses Integral mittels $t=-\log x$ auf die uns vertrautere Form bringen:

$$
\int_{0}^{\infty} e^{-t} t^{s-1} d t=\Gamma(s) \quad(s>0)
$$

Euler interpoliert also in dieser Arbeit die Fakultät unter anderem mithilfe des obigen Integralausdrucks, und erhält so „die Gammafunktion“. Auch wenn Studenten eine solche Erklärung in der Regel widerspruchslos hinnehmen, ist sie problematisch, weil es natürlich beliebig viele Funktionen gibt, die an allen ganzen Stellen vorgegebene Werte annehmen. Dies hat Euler jedenfalls 20 Jahre später, in: De serierum determinatione seu nova methodus inveniendi terminos generales serierum ${ }^{12}$, auch selbst ausgearbeitet und nachdrücklich betont. ${ }^{13}$ Insbesondere löste er dort das Problema 1, den konstanten allgemeinen Term 1 beliebig zu interpolieren, so:

$$
\begin{aligned}
y=1 & +\alpha \sin .2 \pi x+A(\cos .2 \pi x-1) \\
& +\beta \sin .4 \pi x+B(\cos .4 \pi x-1) \\
& +\gamma \sin .6 \pi x+C(\cos .6 \pi x-1)+\text { etc. }
\end{aligned}
$$

\footnotetext{
${ }^{9}$ 20-Pfennig-Briefmarke der DDR 1983, und 130-Rappen-Marke der Schweiz 2007.

${ }^{10}$ E19 (1729/1738; OO, I.14, 1-24).

${ }^{11}$ Die Notationen hier stammen aus dem 19. Jahrhundert: die Grenzen am bestimmten Integralzeichen wurden von Fourier eingeführt und der Buchstabe $\Gamma$ für „die Gammafunktion“ von Legendre.

12 E189 (1750/1753; OO, I.14, 463-515).

${ }^{13}$ Im dritten Abschnitt der Einleitung wird die Botschaft mit Magis mirum videbitur ... angekündigt, die dann lautet: Hinc etsi omnes seriei termini, qui indicibus integris respondent, sunt determinati, intermedios tamen, qui indices habent fractos, infinitis variis modis definire licet, ita ut interpolatio istius seriei maneat indeterminata.
} 
Solche und ähnliche trigonometrische Reihen - vor der Geburt (1768) ihres späteren Namensgebers J.B.J. Fourier - tauchten bekanntlich in der Mitte des 18. Jahrhunderts durch den Streit um die schwingende Saite, d.h. bei der Beschreibung von Lösungen der eindimensionalen Wellengleichung $\frac{\partial^{2} u}{\partial t^{2}}=c^{2} \frac{\partial^{2} u}{\partial x^{2}}$ bei Daniel Bernoulli, Euler und dem jungen Lagrange auf. In dieser querelle hatte Euler den wesentlichen Schritt getan, als Ausgangslage der Saite eine beliebige Kurve anzunehmen, wie sie frei mit der Hand gezeichnet werden kann; aber er bezweifelte, dass die allgemeine Lösung der Gleichung durch Bernoullis trigonometrische Reihen dargestellt werden konnte, da letztere stets ungerade, periodische Funktionen ergaben: Das in Bezug auf analytische Fortsetzung so eigentümliche Verhalten der neuen Reihen war problematisch, lange bevor es bei Fourier und Dirichlet den allgemeinen Funktionsbegriff des 19. Jahrhundert hervorbrachte. ${ }^{14}$

Dass wir in der Mitte des 18. Jahrhunderts „Fourierreihen“ wiedererkennen, bezieht sich auf eine Zukunft, die Euler nicht mehr erlebte. Allerdings mag die Auseinandersetzung mit trigonometrischen Reihen, wie sie insbesondere in der Arbeit E189 aufscheint, Euler zur Einführung seiner ,,inexplikablen Funktionen“ angeregt haben ${ }^{15}$, denen in den Institutiones calculi differentialis zwei Kapitel gewidmet sind, zu deren Beginn es u.a. heisst ${ }^{16}$ :

Ebenso ist der Ausdruck 1.2.3.4. ...x eine inexplikable Funktion von $x$, weil sein Wert, wenn $x$ eine beliebige Zahl ist, nicht nur nicht algebraisch, sondern auch nicht durch irgendeine bestimmte Art transzendenter Grössen ausgedrückt werden kann.

Dieser Wortwahl und Eulers zugehörigen Theorieansätzen war allerdings wenig Erfolg beschieden. Jacques Binet meinte: Il n'est pas facile d'expliquer comment Euler a pu appliquer une telle épithète (inexplicable) à une grandeur dont il connaissait, depuis vingt-cinq ans, l'expression analytique sous forme continue. ${ }^{17}$ Gauß rügte ,diese Erzeugungsart oder Definition“ Eulers als ,durchaus unstatthaft". ${ }^{18}$ Und Euler selbst gelangte am Ende der beiden den „inexplikablen“ Funktionen gewidmeten Kapiteln ${ }^{19}$, nachdem er entwickelt hatte, wie man sie (logarithmisch) ableiten kann, insbesondere zu jenem unendlichen Pro-

\footnotetext{
${ }^{14}$ Siehe etwa den ersten Teil des Berichts [5] und [7], §1. Bei Euler scheinen trigonometrische Reihen zum ersten Mal im Brief an Goldbach vom 4.7.1744 aufzutauchen [10, S. 195-201], in dessen weiterem Verlauf es auch wieder um die „Gammafunktion“ geht, die dort - wie auch in der späteren Arbeit E368 (1765/1769; OO, I.28) - als linea curva, d.i. als Funktionsgraph, zum Ausdruck 1.2.3 ....x angesprochen wird.

${ }^{15}$ Diese Spekulation, die mir naheliegend erscheint, findet sich nicht ausdrücklich in dem Aufsatz [12], dem ich sonst viele Anregungen für diese Passage verdanke. Vgl. auch [17, S. 169-173.]

${ }^{16}$ Es handelt sich um Kapitel XVI und XVII des Zweiten Teils von E212 (vgl. Fussnote 3). Zitat aus §367, S. 769 der Originalausgabe: Simili modo haec expressio 1.2.3.4. ...x, erit functio inexplicabilis ipsius $x$, quoniam si $x$ sit numerus quicunque, eius valor non solum non algebraice, sed ne quidem per ullum certum quantitatum transcendentium genus exprimi potest.

${ }^{17}$ Zitiert nach [14, S. 3.]

${ }^{18}$ Selbstanzeige seiner Abhandlung über die hypergeometrische Reihe, 1812; Werke, Band 3, S. 201. In der Abhandlung selbst macht Gauß die unendliche Unbestimmtheit der Funktion explizit; Werke, Band 3, S. 146. Man kann übrigens fragen, inwieweit Gauß' Arbeit die Algebraische Analysis des 18. Jahrhunderts hinter sich lässt.

${ }^{19}$ Siehe E212, 2. Teil, §402, S. 838 der Originalausgabe. Der Ausdruck ist dort mit $\Lambda$ bezeichnet. Vgl. hierzu auch den oben erwähnten Brief Eulers an Goldbach vom 4.7.1744 [10, S. 197-198].
} 
dukt für $n$ ! zurück ${ }^{20}$, das schon am Anfang der frühen Arbeit E19, wie auch in seinem ersten Brief an Goldbach stand:

$$
\frac{1 \cdot 2^{n}}{1+n} \cdot \frac{2^{1-n} \cdot 3^{n}}{2+n} \cdot \frac{3^{1-n} \cdot 4^{n}}{3+n} \cdot \frac{4^{1-n} \cdot 5^{n}}{4+n} \cdots
$$

Diese Interpolation der Fakultät war damals von Wallis inspiriert gewesen, dessen Identität

$$
\frac{\pi}{4}=\frac{2 \cdot 4}{3 \cdot 3} \cdot \frac{4 \cdot 6}{5 \cdot 5} \cdot \frac{6 \cdot 8}{7 \cdot 7} \cdots
$$

dem jungen Euler den Weg zur Auswertung von $(* *)$ für $n=\frac{1}{2}$ als $\sqrt{\frac{\pi}{4}}$ gewiesen hatte. Freilich ist man mit $(* *)$ noch nicht sofort bei dem Integralausdruck $(*)$.

So bleibt die Frage, wie man Eulers zielstrebige Interpolation in E19 im Rahmen der Geschichte der Algebraischen Analysis methodisch zu bewerten hat - sowohl zur Zeit jener Arbeit als auch im Rückblick von den beiden Kapiteln in E212 aus -, und die analoge Frage stellt sich auch für seine spätere Theorie der inexplikablen Funktionen. Man kann ${ }^{21}$ beide Arbeiten für Beispiele erfolgreicher Praxis der Algebraischen Analysis halten: in E19 wird die Fakultät, die als einfache Zahlenfolge kein Objekt der Algebraischen Analysis ist, durch ein unendliches Produkt, und dann vor allem durch Integralausdrücke, zu einem solchen gemacht; und in E212 werden Reihen, die an sich keine Hinweise zu ihrer Interpolation geben, der Technik des Differenzierens unterworfen. Die Algebraische Analysis ist hier keine Theorie sondern ein Verfahren, das man mit einer gewissen Virtuosität auf natürlich Vorkommendes, im Beispiel auf die Folge der Fakultäten, anwendet in der Meinung, auf diese Weise ihrer Natur näherzukommen.

Diese Einordnung der Mathematik in die Naturphilosophie, die man Euler wohl unterstellen darf, liegt uns recht fern. Wir könnnen nicht darüber hinwegsehen, dass der Erfolg der ersten Arbeit ganz am geschickten Erraten der ,richtigen“ Formel hing, für deren Auswahl ein nachprüfbares Kriterium fehlt. Auch Eulers spätere Ableitung der Formel (**) im Lehrbuch E212 wird uns da nicht zufriedenstellen. So gehen wir spätestens seit Gauß - wenn auch dessen Notation $\Pi(s)$ für das, was wir $\Gamma(s+1)$ schreiben, auf der Müllhalde der Mathematikgeschichte gelandet ist - den umgekehrten Weg und definieren allgemein eine Funktion $\Gamma(s)$, von der sich dann leicht beweisen lässt, dass $\Gamma(n+1)=n$ !, und allgemeiner, dass $s \Gamma(s)=\Gamma(s+1)$ ist. $^{22}$ Eulers Bemühen um eine Analyse der Folge selbst liegt uns fern - es sollte aber als Anliegen des 18. Jahrhunderts nicht in Vergessenheit geraten.

Dieses hier kurz angerissene Beispiel war natürlich sehr speziell. Um ein besseres Gefühl für die Nähe oder Ferne Eulers zu bekommen, schlage ich jetzt einen Weg ein, der stärker von allgemeinen Reflexionen über das 18. Jahrhundert geleitet wird.

\footnotetext{
${ }^{20}$ Auch die Notation $n$ ! stammt erst aus dem 19. Jahrhundert; sie geht auf das Buch Élémens d'arithmétique universelle zurück, das der Mathematik-Magister Christian Kramp $1808 \mathrm{im}$ damals französischen Köln veröffentlichte.

${ }^{21}$ Diese Ansicht wird in der Arbeit [12] vorsichtig nahegelegt.

${ }^{22}$ Die letztere Funktionalgleichung (die alleine die Funktion keineswegs eindeutig festlegt) wird bei Euler explizit für „die“ $n$ ! interpolierende Funktion $y$ von $x$ im einleitenden Abschnitt der späteren Arbeit E368 hingeschrieben; siehe OO, I.28, S. 41-42: ... si abscissae cuicunque $x=p$ conveniat applicata $y=q$, tum abscissae $x=p+1$ respondeat applicata $y=q(p+1)$ usw.
} 


\title{
2 Die Predigtmaschine
}

In Georg Christoph Lichtenbergs (1742-1799) Sudelbüchern kommt Euler einige Male vor; der Eintrag F.1090 von 1780 lautet:

Eulers Maschine Predigten zu spielen, wenn man Worte könnte herausbringen wie Töne, ist ein vortrefflicher Einfall.

Die offensichtliche Ironie angesichts der Idee einer maschinellen Predigt, mit der Lichtenberg hier Eulers Kombination von Forschungsdrang und Frömmigkeit bedenkt, machte mich auf die zugehörigen Stellen bei Euler neugierig. Vermutlich wurde Lichtenbergs Bemerkung durch eine Passage in Eulers Bestseller, den Briefen an eine deutsche Prinzessin veranlasst. ${ }^{23}$ Im 137. Brief ${ }^{24}$ schliesst Euler seine Darstellung der Anfangsgründe der Akustik ab und schreibt im Anschluss an die Erklärung der Lautstärke und Höhe von einfachen Tönen:

\begin{abstract}
Allein es giebt noch einen andern sehr merkwürdigen Unterschied unter den einfachen Tönen, welcher der Aufmerksamkeit der Philosophen scheint entgangen zu seyn. Zwey Töne von gleicher Stärke können mit einerley Ton des Clavieres zusammenstimmen, und demohngeachtet können sie dem Ohre sehr verschieden klingen. Der Ton einer Flöte ist ganz von dem Tone eines Hornes verschieden ... Die wunderbarste Verschiedenheit bemerkt man an der menschlichen Stimme, die das größte Meisterstück des Schöpfers ist ... Geruhen Ew.H. nur über die verschiedenen Vocalen nachzudenken, die der Mund ganz einfach ausspricht oder singet. Wenn man den Buchstaben $a$ singt oder ausspricht, so ist der Ton ganz anders, als wenn man den Buchstaben $e$ oder $o$ oder $i$ oder $u$ oder $a i$ u.s.w. ausspräche oder sänge, auch wenn man bey allen in einerley Tone bleibt. Man kann also die Ursache dieses Unterschiedes nicht in der Schnelligkeit oder der Ordnung der Schwingungen suchen; sie ist uns verborgen, und die Philosophen haben sie bisher noch nicht ergründen können. ... In vielen Orgeln findet man ein Register, das Vox humana (die Menschenstimme) genannt wird; gemeiniglich aber macht sie nur Töne, die den Vocal ai oder ae nachahmen. Ich zweifle nicht, daß man mit einigen Veränderungen auch die übrigen Vokalen $a, e, i, o, u$, würde herausbringen können; aber all dieses würde noch nicht hinreichen, ein einziges Wort der menschlichen Stimme nachzuahmen; denn wie sollte man die Consonanten mit ihnen verbinden, die so viele Modificationen der Vocalen sind? Unser
\end{abstract}

\footnotetext{
${ }^{23}$ Die Lettres à une Princesse d'Allemagne sur divers sujets de physique \& de philosophie, E343, E344, E417 (St. Petersburg 1768-1772; OO III.11, III.12) sind von 1760-1762 datiert, waren an die junge Tochter des mit Euler bekannten Markgrafen von Brandenburg-Schwedt gerichtet und in der Bildungssprache Französisch abgefasst. Die erste deutsche Übersetzung, vermutlich von Euler selbst besorgt, erschien ab 1769 in Leipzig bei J.F. Junius. Der verlegerische Erfolg dieser Naturphilosophie in allgemeinverständlicher Darstellung ist kaum zu überschätzen. Eneströms Verzeichnis der Werke Eulers zählt 111 verschiedene Ausgaben. In einem Brief vom 26.7.1835 bittet Franz Liszt seine Mutter, ihm seine liebsten Bücher (sorgfältig in Wäsche für die Gräfin d'Agoult eingepackt) nachzusenden. In Liszts kurzer Liste stehen Eulers Lettres gleich hinter Shakespeare und Lord Byron und vor Madame d'Agoults Bibel; siehe [21, S. 82].

${ }^{24}$ Der Brief trägt das Datum 16.6.1761. Deutsches Zitat nach der Erstübersetzung (1769-1773), nachgedruckt Braunschweig (Vieweg) 1986. Für das vollständige Originalzitat siehe Anhang 4.1 unten.
} 
Mund ist so bewundernswürdig eingerichtet, daß es uns unmöglich ist, den Mechanismus, der zu diesem so gemeinen Gebrauche desselben gehört, zu ergründen. ... Ohne Zweifel wäre das eine von den wichtigsten Entdeckungen, wenn man eine Maschine erfünde, die alle Töne unsrer Wörter mit all ihren Artikulationen aussprechen könnte. Wenn man jemals mit einer solchen Maschine zustande käme, und sie durch gewisse Orgel- oder ClavierTasten alle Wörter könnte aussprechen lassen; so würde alle Welt mit Recht erstaunt seyn, eine Maschine ganze Reden hersagen zu hören, die man mit der größten Anmuth würde vergesellschaften können. Die Prediger und Redner, deren Stimme nicht stark oder nicht angenehm genug wäre, könnten alsdann ihre Predigten und Reden auf einer solchen Maschine spielen ... Die Sache scheint mir nicht unmöglich zu sein.

Zwei Tage nach diesem Brief an die Prinzessin, am 18.8.1761, schrieb Euler einen Brief an seinen schweizerischen Landsmann ${ }^{25}$ Johann Heinrich Lambert, in dem es sich grösstenteils um die Mondbewegung dreht, an dessen Ende Euler aber unser Thema aufgreift $^{26}$ :

Bisher hat man den Schall immer nur in Bezug auf die Tonhöhe untersucht, aber man bemerkt doch so viele andere Unterschiede. So sind z.B. zwei Töne, von denen einer den Vokal $a$ ausspricht, der andere $e$, oder $i$, oder $o$, oder $u$, verschieden, auch wenn sie auf der gleichen Tonhöhe sind. Und ich weiß nicht, ob sich schon jemand die Mühe gemacht hat, zu untersuchen, worin der Unterschied dieser Töne besteht und wodurch sich die Erregungen der Luft unterscheiden. Könnte man nicht Maschinen herstellen, die die Vokale klar aussprechen, was unweigerlich einen guten Effekt machen würde. Vielleicht käme man schließlich dahin, sie auch mit Konsonanten zu kombinieren, und die Mechanik schließlich so weit zu treiben, daß man eine Maschine baut, die deutlich ganze Worte ausspricht, und sogar Ansprachen hält. Das wäre hervorragend für alle Prediger, deren Stimme nicht hinreichend kräftig oder wohlklingend ist. In diesen Forschungen müßte man nur der Natur und dem Organismus unserer Sprache folgen.

Das Thema der Analyse des Sprechens setzt sich im Briefwechsel bei Euler und Lambert weiter fort - siehe die Anhänge 4.3 bis 4.7 unten. Die eben zitierte Stelle aus Eulers Brief an Lambert vom August 1761 zeigt der Herausgeber Karl Bopp in einer kurzen Zusammenfassung dieses Schreibens durch die Worte: „Vorahnung des Phonographen“ an. ${ }^{27}$ Wir sehen heute sofort, dass das nicht stimmt: Euler beschreibt ja, dass er Stimmen nicht auf-

\footnotetext{
${ }^{25}$ Lambert (1728-1777) nannte sich Mulhousino helveticus; seine Geburtsstadt Mulhouse war seinerzeit unabhängig aber mit der Schweiz verbündet. Erst nach der Revolution, am 15.3.1798, gliederte sie sich Frankreich ein.

${ }^{26}$ Meine Übersetzung aus [11, S. 27]; siehe Anhang 4.2 unten. Das Original dieses Briefes, das ich zur Kontrolle beigezogen habe, befindet sich im Archiv der Universitätsbibliothek Basel, wohin es in den 1930er Jahren durch den ebenso glücklichen wie ungewöhnlichen Verkauf eines bedeutenden Korpus von Bernoulliana von Gotha aus gelangt ist.

${ }^{27}[11$, S. 5]. Bopps Zusammenfassungen sind unverändert en bloc in den Übersichtsband OO IVA.1, Descriptio commercii epistolici, Hrsg. Juškevič, Smirnow und Habicht, S. 245, Nr. 1413, übernommen worden.
} 
zeichnen sondern maschinell erzeugen möchte. Im 137. Brief an die Prinzessin - den Bopp nicht erwähnt - wurde Euler ja noch genauer und entwarf das Bild des wenig sprachgewaltigen Predigers der Zukunft, der seine donnernde Botschaft auf einer Art Sprachorgel mit Tasten und Registern der gebannten Gemeinde vorspielt. Im Gegensatz zum Phonographen ist Eulers Predigtmaschine ein Instrument, auf dem der Spieler Texte weder aufnimmt noch abspielt, sondern aktual spielt. Euler hatte keine „Vorahnung des Phonographen“. Vielmehr projiziert umgekehrt Karl Bopp mit seiner Inhaltsangabe ein technisches Gerät, das in den 1920er Jahren eine stolze Errungenschaft der Technik darstellte und damals auch ganze Forschungszweige wie etwa die Musikethnologie inspirierte ${ }^{28}$, in einen Text des 18. Jahrhunderts hinein.

Diese „Vorahnung des Phonographen“ ist nicht nur technisch unzutreffend, sondern sie verleitet auch dazu, ein Bild Eulers als eines Wissenschaftlers zu entwerfen, der, wie man so sagt, ,,seiner Zeit voraus war" und dessen Texte man deshalb in trügerischer Nähe zu unserer Jetztzeit lesen sollte. Ein solches Ansinnen ist allemal mit Vorsicht zu geniessen; im Falle Eulers führt es m.E. völlig in die Irre. ${ }^{29}$ Denn Leonhard Euler war ganz und gar ein Kind seiner Zeit - natürlich nach Begabung und Leistungsfähigkeit ein Ausnahmekind; aber mit diesen Fähigkeiten verlieh er eben seinem 18. Jahrhundert in Büchern und Aufsätzen Ausdruck. Zwar konterkariert Eulers Frömmigkeit die dominierenden philosophischen Tendenzen jener Zeit. Aber so sehr dieser Zug Euler mitunter dazu verleitete, seine akademische Macht zu missbrauchen ${ }^{30}$, so wenig wird man andererseits diese seltene Frömmigkeit im Zeitalter der Aufklärung als Zeichen dafür nehmen wollen, dass Euler „seiner Zeit voraus" war.

In der Tat ist Eulers Idee einer Sprechmaschine, und die Art wie er sie vorbringt, sehr typisch für die Rolle, welche Maschinen im 18. Jahrhundert zugemessen wurde. Euler fragte, ganz Naturphilosoph, einerseits nach der physikalischen Natur der Klangfarbe im Allgemeinen und andererseits nach der Art und Weise, wie wir beim Sprechen oder Singen verschiedene Klänge erzeugen. Eine Theorie der Klangfarbe durch das Spektrum der Obertöne stand ihm nicht zur Verfügung; die oben erwähnten ersten Anfänge der Fourieranalyse seiner Zeit konnte er daher nicht für eine mathematische Behandlung der beschriebenen Vielfalt akustischer Phänomene fruchtbar machen. ${ }^{31}$ Die Idee der Sprechmaschi-

\footnotetext{
${ }^{28}$ Béla Bartók z.B. bereiste Anfang der 1930er Jahre den Balkan, Nordafrika und die Türkei und nahm mit dem Edisonschen Phonographen Volksgesänge auf.

${ }^{29}$ Ein anderer Fall fragwürdiger Rückprojektion moderner Auffassungen, den wir hier nicht weiter diskutieren, tritt in dem 2004 erschienenen Band OO III.10 der Euler Gesamtausgabe auf, wenn D. Speiser und P. Radelet-de Grave im Kommentar zu E842: Anleitung zur Naturlehre auf p. CXV schreiben: „Thus Euler was the first to conceive and to imagine a unified field theory, an idea that stands today in the centre of the physicist's aim." - Vgl. demgegenüber das Euler-Kapitel in [22], mit Hinweis auf die angekündigte Herausgabe durch Andreas Kleinert des Briefwechsels zwischen Euler und Le Sage.

${ }^{30}$ Siehe die neuere Studie [15]. Im zweiten Band beschreibt diese Autorin sehr genau, wie die Berliner Akademie-Affaire von 1752-1753 - in welcher der Präsident Pierre-Louis Moreau de Maupertuis, Euler und sein schweizer Landsmann, der Philosoph Johann Bernhard Merian, den Wolffianer Samuel König massregelten - eine regelrechte öffentliche Debatte auslöste, die schon vor Voltaires Eingreifen europäische Dimensionen hatte. Unser heutiges Unbehagen an der Arroganz jener akademischen Machtausübung passt demnach sehr gut zu den historischen Ereignissen und wird durch Eulers weltanschauliche Motive schon deshalb nicht beschwichtigt, weil er aus seinem Verhalten verschiedene handfeste Vorteile zog; siehe insbesondere [15, II, S. 568].

${ }^{31}$ Es ist instruktiv, dieser Situation Eulers die kompositorische Behandlung derselben Phänomene gegenüberzustellen, die Karlheinz Stockhausen in seinem Stück Stimmung (Nr. 24 von 1968) vorgelegt hat. Dort
} 
ne tritt daher - in den Briefen an eine deutsche Prinzessin bezeichnender Weise sowohl mit dem Wort 'Entdeckung' als auch mit 'erfinden' 32 gepaart - als Lösung des Problems auf, das mathematisch unverstandene, von der belebten Natur aufgeworfene physikalische Phänomen der Klangfarbe in dem besonders komplizierten Falle der menschlichen Artikulationen durch möglichst genaue mechanische Reproduktion des menschlichen Sprechapparats zu analysieren.

Die Erforschung durch Konstruktion einer Maschine nahm für Euler anscheinend eine Mittelstellung im Gesamtspektrum wissenschaftlicher Methoden ein. Dabei stehen auf der einen Seite der Skala solche Naturprozesse, die sich theoretisch, d.h. mathematisch beschreiben lassen. Dazu gehört z.B. die Mechanik, oder die Akustik, soweit sie nur die Ausbreitung der Töne und deren Grundfrequenzen behandelt. Dazu gehört aber auch, ganz an diesem Ende der Skala, die gesamte mathematische Analysis selbst, insofern sie für Euler eine theoretische Methode zur Entdeckung und genaueren Untersuchung naturgegebener Grössen und ihrer Verhältnisse ist - im speziellen Beispiel des Abschnitts 1. oben ging es um das rechte Verstehen gegebener Folgen. Ein solcher Gegenstandsbereich in der äusseren Wirklichkeit ermöglicht den Bau präziser Maschinen; aber da er der mathematischen Theorie zugänglich ist, haben diese Maschinen selbst kein vorrangiges Erkenntnisziel, sondern ihre Theorie muss möglichst genau mathematisch ausgearbeitet werden. So hat sich Euler wiederholt für eine bessere (z.B. die Energiebilanz einbeziehende) mathematische Behandlung alltäglicher mechanischer Maschinen eingesetzt. ${ }^{33}$

Am anderen Ende der Skala stehen jene Naturphänomene, die nicht nur nicht durchgreifend mathematisiert werden können, sondern für die - im Gegensatz zur menschlichen Sprache - auch keine Hoffnung besteht, sie maschinell zu synthetisieren und dadurch unserem beherrschbaren Wissen einzuverleiben. Ein Beispiel hierfür, das in Anbetracht von Eulers Sorgen um sein Augenlicht wohl auch einen sehr persönlichen Aspekt hat, ist die Behandlung des menschlichen Auges in den Briefen Nr. 41-44 an die deutsche Prinzessin. Gleich zu Anfang, und ebenso am Ende dieser Passage, betont Euler, dass auch der geschicksteste Künstler niemals ein Sehorgan konstruieren könnte, das nicht unendlich viel schlechter als unser Auge wäre. In diesen Fällen, die weder der Mathematik noch der Maschinenkunst zugänglich sind, öffnet Euler der Verehrung des Schöpfers noch weiter die Tore als das in den Briefen an die Prinzessin ohnehin oft geschieht: das Rätsel z.B., wie das Bild auf der Retina von der Seele angeschaut werden kann, wird zur Widerlegung der Materialisten erhoben, und im 43. Brief wird die Notwendigkeit der Materialkombination in der Augenlinse gewürdigt. ${ }^{34}$

wird das theoretische Grundverständnis der Klangfarbe durch die diskrete Folge der Obertöne einfach vorausgesetzt und in der Notation der Partitur und den beigegebenen Erläuterungen für die Sänger explizit benutzt.

${ }^{32}$ Im Französischen découverte und construire.

${ }^{33}$ Von den einschlägigen Arbeiten E96, E194, E277 beachte man besonders die sehr ausführliche Einleitung zu E194, De machinis in genere (1746/1750; OO II.17), die ein glühendes Plädoyer für die Zusammenführung von Ingenieurwissenschaften und höherer Analysis ist. Für das Verständnis von Eulers Methodik scheint mir das wichtig, auch wenn die erwähnten Arbeiten heutige Ingenieurwissenschaftler viel weniger interessieren als etwa Eulers Arbeiten über Zahnräder - siehe den etwas gelangweilten Kommentar in OO, II.17, S. XLV-LII.

${ }^{34}$ Siehe z.B. den Satz, der von der Unmöglichkeit der Theorie zur Unmöglichkeit der Maschine fortschreitet, am Anfang des 41. Briefes (OO III.11, S. 94): „Nous reconnoîtrons dans la structure des yeux, des perfections que l'esprit le plus éclairé ne sauroit jamais approfondir; et le plus habile artiste ne sauroit jamais fabriquer une machine de cette espèce, qui ne soit infiniment au-dessous de tout ce que nous découvrons dans les yeux; quand 
Maschinen galten damals vielen Biowissenschaftlern als Mittel der Erkenntnis. Gleichzeitig reichte ihre Bedeutung durch die öffentliche Zurschaustellung durchaus wissenschaftlich motivierter Maschinen weit über den engen Zirkel der Experten hinaus, und auch dieser zweite Aspekt ist in Eulers Briefen greifbar, wenn er nicht nur die deutsche Prinzessin sondern auch Lambert besonders auf den „guten Effekt" hinweist, den der öffentliche Gebrauch einer Predigtmaschine machen würde. Der seit Ende der 1730er Jahre berühmteste Bastler und Vorführer bemerkenswerter Verstehens-Maschinen war Jacques Vaucanson (1709-1782), dessen mechanischer Flötenspieler (1737), dessen Trommler, und vor allem dessen Canard digérateur (1739) - die Körner pickende und verdauende sowie flügelschlagende Ente aus über 400 beweglichen Einzelteilen - ihn zu einer europäischen Berühmtheit machten, die Friedrich II. vergeblich nach Berlin zu ziehen versuchte. ${ }^{35}$ Bei der Vorführung des Canard digérateur wurde auch der innere Mechanismus, insbesondere der flexible Gummidarm gezeigt. So überrascht Vaucansons Plan von 1741 nicht, einen anatomischen Automaten zu konstruieren, der medizinische Theorien über die Körperfunktionen testen und zur Ausbildung der Mediziner herangezogen werden sollte. Der Plan wurde nicht realisiert; stattdessen beschäftigte sich Vaucanson im Regierungsauftrag mit der Mechanisierung der Seidenherstellung und -verarbeitung - stiess dort freilich auf Widerstand der Beschäftigten. Sein frühkapitalistisch-optimistisches Konzept beruhte auf der Überzeugung, dass bei der maschinellen Seidentuchherstellung die Qualität des Tuches von den handwerklichen Fähigkeiten des Arbeiters ganz unabhängig sein würde. Hier besteht eine Analogie zu Eulers Empfehlung der Sprechmaschine für Prediger mit unzureichender Stimme. 1757 erhielt Vaucanson die Stelle eines Mechanikers an der Pariser Akademie gegen seinen Gegenkandidaten Denis Diderot. ${ }^{36}$

In der Nachfolge und Radikalisierung seines Lehrmeisters Herman Boerhaave verlieh der Mediziner und Philosophe Julien Offray de La Mettrie - den Euler sich wohl hütete zu erwähnen, während Friedrich II. nach dem plötzlichen Tod (1751) des 43-jährigen eine Éloge vor der Berliner Académie hielt - in seinem flott geschriebenen Buch L'Homme Machine von 1747 einer zum materialistischen Credo gesteigerten naturwissenschaftlichen Medizin Ausdruck. ${ }^{37}$ Eine oft zitierte Passage daraus, von der ich freilich nicht weiss, ob Euler sie kannte, lautet ${ }^{38}$ :

[Der Mensch] verhält sich zum Affen, zu den geistigsten Tieren, wie Huygens Planetenuhr zu einer Taschenuhr von Julien le Roi. Wenn es mehr Instrumente, Räderwerke, Federn brauchte, um die Planetenbewegungen anzuzeigen als um die Stunden anzugeben; wenn Vaucanson mehr Kunstfertigkeit brauchte, um seinen Flöter zu machen als für seine Ente, so hätte er noch mehr aufwenden müssen, um einen Sprecher zu machen - eine Maschine, die nicht mehr

même nous lui accorderions le pouvoir de former la matière à son gré, et le plus haut degré de pénétration dont un homme peut être susceptible.“ Und zusammenfassend am Anfang des 44. Briefes (OO III.11, S. 100): „L'oeil surpasse donc infiniment toutes les machines que l'adresse humaine est capable de produire.“

${ }^{35}$ Siehe [15], Band II, S. 531.

${ }^{36}$ Ich nutze hier und im Folgenden Simon Schaffers Analyse in [26], um Eulers Idee einzuordnen. Vgl. auch [3].

${ }^{37}$ Trotz gelegentlicher Reserven habe ich mich bei der Lektüre La Mettries von Kathleen Wellmans mit grosser Sympathie für den Freigeist geschriebenem Buch [30] orientieren lassen.

${ }^{38}$ Meine Übersetzung; siehe das Original im Anhang 4.8 unten. 
als unmöglich angesehen werden kann, besonders nicht in den Händen eines neuen Prometheus.

Mehr als 20 Jahre später, im 69. Jahre jenes prometheischen Jahrhunderts, führte Baron Wolfgang von Kempelen (1734-1804), Hofkammerrat unter Maria Theresia, in Wien zum ersten Mal seinen schachspielenden Türken vor. Dieser war zwar insofern ein Betrug, als darin ein Mann versteckt war, der sich die Spielzüge ausdachte und dann mittels des Mechanismus der Türkenfigur ausführte. ${ }^{39}$ Aber auch dieser Betrug belegt noch das spezifische Interesse der damaligen Zeit an mechanischen Nachahmungen des Lebens. Die (vermeintliche) Maschine, die sogar die Intelligenz des Schachspielers nachahmen sollte, war als ultimative Antwort auf Vaucansons Schaustücke gedacht, die ausserdem andere Konkurrenten an Maria Theresias Hof, wie z.B. den Hofmechaniker Friedrich von Knauss (1724-1789), aus dem Felde schlagen sollte.

Es gibt zwei Berührungspunkte zwischen Leonhard Euler und Wolfgang von Kempelen. Der erste ist Eulers Idee der Sprechmaschine. Baron von Kempelen war nämlich an sich keineswegs ein betrügerischer Schausteller. Neben dem - freilich seinerzeit nur durch Betrug erreichbaren - Ziele, das Nachdenken eines Schachspielers maschinell nachzuahmen, arbeitete er ernsthaft und erfolgreich an der mechanischen Nachahmung der anderen Gabe, die vielleicht den Menschen vom Tier unterscheidet: der menschlichen Sprache. Diese Arbeiten gipfelten einerseits in seinem Buch Mechanismus der menschlichen Sprache nebst Beschreibung einer sprechenden Maschine ${ }^{40}$ und andererseits in verschiedenen Prototypen dieses Apparats, von denen einer noch heute im Deutschen Museum in München zu sehen ist, und der anscheinend lateinische, italienische und französische Wörter zu Gehör bringen konnte.

Aber von Kempelen war hiermit nicht der erste. Sein älterer Konkurrent am Hofe, Friedrich von Knauss, hatte schon 1770 vier „sprechende Köpfe“ konstruiert, denen freilich kein durchschlagender Erfolg beschieden war: 1779 - bevor von Kempelens Arbeiten hinreichend fortgeschritten waren - entsann sich Euler nämlich seiner alten Idee und liess als Preisaufgabe der St. Petersburger Akademie die beiden Fragen ausschreiben: 1. die Natur des Unterschieds der Vokale zu erklären, und 2. eine Maschine nach dem Vorbild der Vox humana Pfeifen der Orgeln zu konstruieren, die die Vokale richtig aussprechen kann. Der Preis ging an den Physiologen Christian Kratzenstein, der sich eng an die menschliche Vokalbildung und den Ausbau der Vox humana Pfeifen hielt, so wie Euler es zuerst konzipiert hatte. ${ }^{41}$

Soviel zu Eulers Idee einer Predigtmaschine als charakteristisches Projekt des 18. Jahrhunderts. Der andere Berührungspunkt zwischen Euler und von Kempelen ergibt sich aus dem vermeintlichen Schachautomaten. Bei den öffentlichen Auftritten des „Türken“ spielte die vorgebliche Maschine vor allem Schachpartien gegen Mitglieder des Publikums. Von Kempelen hatte aber auch andere Nummern im Programm: Im November 1784 z.B. zeigte der „Türke“ bei seinem Auftritt auf der Leipziger Messe, dass er, von einem beliebig aus dem Publikum benannten Feld des Schachbretts ausgehend, einen Springer jedes

\footnotetext{
${ }^{39}$ Siehe [25]; vgl. [26].

${ }^{40}$ Mit 27 Kupfertafeln, verlegt in Wien bei J.V. Degen 1791.

${ }^{41}$ Siehe [28].
} 
Feld des Bretts genau einmal besuchen lassen konnte. ${ }^{42}$ Dieses Problem hatte Euler in seiner Arbeit E309 ${ }^{43}$ in den Berliner Mémoires de l'Académie Royale des Sciences et Belles Lettres gelöst und gehörig analysiert, obgleich auf solche Untersuchungen ,sonsten die Analysis keinen Einfluss zu haben scheinen möchte“. In dieser Arbeit druckte er frühzeitig einen Weg ab, der den Springer nicht nur genau einmal über jedes Feld führt, sondern der ausserdem ,in se rediens ist“ ${ }^{44}$, d.h. zu einem Endpunkt führt, von dem aus man auf den Anfangspunkt zurückspringen kann. So konnten die akademisch geweihten Springerzüge des „Türken“ dem staunenden Publikum seinerzeit als gelungenes Zusammenkommen von wissenschaftlicher Analyse und Maschinenleistung präsentiert werde, zumal Eulers Arbeit ihrerseits mit einer leutseligen Schilderung der Umstände beginnt, unter denen er selbst das Problem in einer Gesellschaft kennen und als schwer schätzen lernte. ${ }^{45}$

\section{Algebra und Zahlentheorie}

Eulers Analyse der Springerwege in E309 strahlt nicht nur wegen ihrer erzählerischen Einbettung eine unmittelbare Nähe aus; sondern auch, weil Euler beim Schildern dieses vorbildlosen Aufspleissens und Wiederzusammenfügens von Wegeteilen sein ganzes pädagogisches Geschick aufbietet. Es geht einem hier so wie oft in der Vollständigen Anleitung zur Algebra ${ }^{46}$, wo der Autor ebenfalls den Gegenstand in kleinen Schritten, mit häufig wiederholten Präzisierungen für den Leser aufbaut. Im Falle der Algebra, z.B. bei der Auflösung von Gleichungen, bringen wir den so angenehm entwickelten Stoff spontan mit unserem Vorwissen in Einklang. Damit kommen wir diesem erfolgreichen Lehrbuch nicht unbedingt näher. Denn Eulers häufigstes Wort, wenn er über Algebra redet, ist „Kunstgriffe“. Die „Analytik oder Algebra“, wie er sie selbst nennt, wirkt bei ihm eher wie eine reichhaltige, für weite Teile der Mathematik grundlegende Trickkiste, denn wie eine systematische Theorie.

Zu Eulers Analyse des Schachproblems gibt es unterschiedliche Ansichten: ob es sich um Freizeitmathematik ${ }^{47}$ handelt oder um den Beginn der kombinatorischen Topologie. ${ }^{48}$ Das hat wenig Bedeutung, solange Eulers entscheidender Anspruch nicht in Vergessenheit gerät: in dieser Arbeit eine systematische analyse dieses Problems entwickelt zu haben, welche allein die Publikation dieser Materie rechtfertigt. Euler unterstreicht in E309 seinen Erfolg, eine auf den ersten Blick nur dem unsystematischen Probieren zugängliche Aufgabe vollständig gelöst zu haben. Er beherrscht die sehr grosse, wenn auch endliche Anzahl der möglichen Springerwege. So steht diese Analyse mindestens auf dem theoretischen Niveau der Algebra, die rhetorisch eher erniedrigt wird.

\footnotetext{
${ }^{42}$ Siehe [26, S. 154]. Vgl. [31].

${ }^{43}$ Solution d'une question curieuse qui ne paroit soumise à aucune analyse (Berlin 1758/1766; OO I.7).

${ }^{44}$ Die letzten beiden Zitate aus Eulers Brief an Goldbach vom 26.4.1757, [10, S. 394].

${ }^{45}$ Allerdings hätte z.B. Eulers rekurrenter Weg wohl auch schon im 18. Jahrhundert durch einen Uhrwerkmechanismus relativ leicht umgesetzt werden können - eben weil er weder mit der Fähigkeit erfolgreich Schach zu spielen noch, wenn man ihn einmal kennt, Eulers scharfsinnige Analysen der transformations gegebener Wege in $\mathbf{E 3 0 9}$ benutzt. Aber diese Überlegung geht an der schaustellerischen Wirklichkeit des „Türken“ vorbei.

${ }^{46}$ Zwei Bände, E387, E 388 (St. Petersburg 1771; OO, I.1). Vor dieser deutschen Erstauflage war eine russische Fassung erschienen.

${ }^{47}$ So der Kommentar zu E309 im „Euler Archive“, http:// www.math.dartmouth.edu/ euler/

${ }^{48}$ So [10, S. 394, Fussnote 2].
} 
Bei der Einordnung von Eulers Arbeiten zur Zahlentheorie stellen sich kompliziertere Fragen. Bekanntlich gehörte Euler, angestossen durch Christian Goldbach, zu den wenigen Mathematikern des 18. Jahrhunderts, die sich intensiv mit zahlentheoretischen Fragen beschäftigten, wie sie vor allem Fermat der Nachwelt hinterlassen hatte. Dazu gehören in erster Linie Probleme der (Un-)lösbarkeit gewisser diophantischer Gleichungen. Für diese „unbestimmte Analytik“ hat Euler im letzten Abschnitt seiner Vollständigen Anleitung zur Algebra ${ }^{49}$ zwar ein erstes Kompendium geschrieben, welches in der französischen Übersertzung durch Lagranges Zusätze zu Eulers Elementen der Algebra, Unbestimmte Analytik ${ }^{50}$ noch kongenial ergänzt wurde. Aber erstens ist bei Euler der Umfang und Status dieses Gebiets innerhalb der Algebra, und im Verhältnis zur übrigen algebraischen Analysis, nicht ganz klar. In charakteristischem understatement schreibt Euler im $§ 2$ dieses Abschnitts, nachdem er die hier zusammengefassten Probleme als unbestimmte (weniger Gleichungen als Unbekannte), zu denen Ganzzahligkeitsbedingungen hinzugefügt werden, umrissen und schwierige Fälle angekündigt hat: „,Daher dieser Theil der Analytik oft ganz besondere Kunstgriffe erfordert, und sehr zur Belehrung der Anfänger sowie zur Ausbildung ihrer Fertigkeit im Rechnen dient.“ So unspektakulär werden also hier z.B. die Beweise der Fermatschen Vermutung für den Exponenten 3 (\$243) und 4 (\$205) eingeleitet.

Zweitens und vor allem stehen wir jener Zeit, als Adrien-Marie Legendre in der Einleitung $\mathrm{zu}$ seinem Essai sur la théorie des nombres ${ }^{51}$ Zahlentheorie mit unbestimmter Analytik identifizieren konnte, zu fern, um nicht nach anderen Arbeiten Eulers zu fragen, die wir heute der Zahlentheorie assoziieren würden ${ }^{52}$, z.B. nach Eulers Behandlung der Riemannschen Zetafunktion. ${ }^{53}$ Das ist immer dann anachronistisch, wenn es sich um analytische Arbeiten handelt, die für Euler einfach zur algebraischen Analysis gehörten ${ }^{54}$, deren zahlentheoretische Ehren aber aus späterer Zeit stammen, als Cauchys oder Weierstraß' Analysis den Ton angaben - vgl. Abschnitt 1 oben. Diese Zeitverrückung müsste uns zögern lassen, in solchen Fällen von Zahlentheorie bei Euler zu reden.

Die bisher eingehendste und einsichtsvollste Gesamtstudie über Eulers ,,zahlentheoretische Arbeiten“ stammt von André Weil. ${ }^{55}$ Weil zeichnet nicht nur eindrucksvoll die Fülle und zeitliche Struktur der ausgewählten Arbeiten Eulers nach ${ }^{56}$, sondern ermöglicht dem Eingeweihten, der die Anspielungen auf die heutige Sichtweise auf die Probleme versteht

\footnotetext{
${ }^{49}$ Zweiter Band, d.i. E 388, Zweiter Abschnitt: „Von der unbestimmten Analytik“.

${ }^{50}$ Élémens d'algèbre, par Léonard Euler, traduits de l'allemand avec des notes et des additions par Jean Bernoulli et Joseph-Louis Lagrange. Lyon (J.-M. Bruyset). 1784 ${ }^{1}$; an III $^{2}$ [= 1794-95].

${ }^{51}$ Erste Auflage Paris (Duprat) 1798.

${ }^{52}$ In [16] wird der Wandel des disziplinären Umfelds der Zahlentheorie über die erste Hälfte des 19. Jahrhunderts im einzelnen nachgezeichnet. Für die Ausgangssituation nach Euler und Lagrange, die Legendre zur Einordnung seines Essai veranlasste, siehe [16, §2].

${ }^{53}$ Für eine formelorientierte Übersicht über die damit angesprochenen Arbeiten Eulers, siehe [1].

${ }^{54}$ Also z.B. alle bei Ayoub aufgeführten Arbeiten.

55 [29, chap. III]. Frz. Fassung dieses Kapitels in [6, S. 111-134].

${ }^{56}$ In [29, chap. III, §V] zählt der Autor die „dramatis personae“ auf, die er zu Eulers zahlentheoretischen Arbeiten zusammenfasst: (a) Fermat's theorem, the multiplicative group of integers modulo $N$, and the beginning of group theory; (b) Sums of squares and „elementary“ quadratic forms; (c) Diophantine equations of degree 2; (d) Diophantine equations of genus 1, and others; (e) Elliptic integrals; (f) Continued fractions; (g) Summation of $\zeta(2 n)$ and related series; (h) Partitio numerorum and formal power- series; (i) Prime divisors of quadratic forms; (j) Large primes [insbesondere über die numeri idonei]. Aus heute aktueller Sicht müsste man im Sinne Weils auch die Multizetawerte $\zeta(r, s)=\sum_{m>n>0} \frac{1}{m^{r} n^{s}}$ hervorheben, von denen einige bei Euler im Brief an
} 
und einzuordnen weiss, eine privilegierte Lesart von Eulers Arbeiten. Die Breite der Arbeiten freilich, die Weil zu Eulers Zahlentheorie zusammenfasst, mag insofern erstaunen, als er sich bei anderen Anlässen durchaus restriktiver zeigen konnte ${ }^{57}$ :

When I smell number-theory I think I know it, and when I smell something else, I think I know it too. For instance, there is a subject in mathematics (it's a perfectly good and valid subject and it's perfectly good mathematics) which is misleadingly called Analytic Number Theory. In a sense, it was born with Riemann who was definitely not a number theorist; it was carried on, among others, by Hadamard, and later by Hardy, who were also not number- theorists (I knew Hadamard well); and to the best of my understanding, analytic number theory is not number theory. What characterizes it as analysis ... is that it deals with inequalities and asymptotic evaluations; this, to me, characterizes it as being something else than number theory.

Wie dem auch sei, jedenfalls hatte Weil ein waches historisches Gewissen. So kommt es, dass er zumindest in Nebensätzen oder speziellen Formulierungen den Anachronismus der Sichtweise, von der er sich leiten lässt, doch nicht aus dem Auge verliert. Das Ergebnis ist, dass er streckenweise ausdrücklich einen Euler bescheibt, von dem er gleichzeitig sagt, dass es ihn gar nicht gab. Dazu nur ein Beispiel ${ }^{58}$ : „What we now call 'elliptic curves' (i.e., algebraic curves of genus 1) were considered by Euler under two quite different aspects, without his ever showing awareness of the connection between them, or rather of their substantial identity." Weil meint hier einerseits den diophantischen Aspekt (Abstiegsmethode) und andererseits den Aspekt der elliptischen Integrale. Einmal abgesehen von der Tatsache, dass der hier bemühte Begriff der elliptischen Kurven erst in der zweiten Hälfte des 19. Jahrhunderts entstand, wuchsen auch die beiden erwähnten Gesichtspunkte, zwar früher, also ohne elliptische Kurven, aber doch erst zu einer Zeit zusammen, die nicht nur nach Eulers Tod, sondern sogar erst nach einem Vierteljahrhundert Wirkungsgeschichte von Gauß' Disquisitiones Arithmeticae (erschienen 1801) reif war: Als C.G.J. Jacobi in dem kleinen Artikel [18] für den Gebrauch elliptischer und abelscher Integrale zum Auflösen diophantischer Gleichungen warb, stand das eigentümliche Forschungsfeld, das wir in [16, §3], Arithmetische Algebraische Analysis genannt haben, in seiner ersten Blüte. Seit den 1820er Jahren und bis in die Mitte des 19. Jahrhunderts hinein, suchte der neue, von Gauß angestossene arithmetische Impuls die Vermittlung mit analytischen Methoden, deren eine Quelle Eulers Leistungen auf dem Gebiete der elliptischen Integrale, deren andere Quelle Fouriers trigonometrische Reihen waren. Die spätere Vermittlung von zahlentheoretischen Gleichungen und Integralen durch den Begriff der elliptischen Kurve hingegen wurde erst in der Gründungsurkunde der arithmetischen algebraischen Geometrie: in dem Aufsatz [24] besiegelt. ${ }^{59}$

Goldbach vom 24.12.1742 und in der Arbeit E477, Meditationes circa singulare serierum genus (1771/1776; OO I. 15) vorkommen.

${ }^{57}$ Siehe A. Weil, OEuvres Scientifiques / Collected Papers, vol. III, S. 280.

58 [29, S. 242].

${ }^{59} \mathrm{Vgl}$. [27]. 


\section{Anhänge: Original-Zitate}

4.1. Französisches Original der Stelle aus Eulers 137. Brief an die deutsche Prinzessin ${ }^{60}$ :

Mais parmi les sons simples il y a même encore une différence très remarquable, qui semble être échapée à l'attention des Philosophes. Deux sons peuvent être également forts et d'accord avec le même son du clavecin, et malgré cela ils peuvent être très différens à l'oreille. Le son d'une flûte est tout à fait différent de celui d'un cor; quoique tous les deux conviennent avec le même ton du clavecin et soient également forts. C'est ainsi que chaque son tient quelque chose de l'isntrument qui le rend; et on ne sauroit presque dire en quoi cette qualité consiste; aussi la même corde rend-elle des sons différens à cet égard, selon qu'elle est frappée, touchée ou pincée; et V.A. sait très bien distinguer les sons des cors, des flûtes, et d'autres instrumens.

La plus admirable diversité s'observe dans la voix humaine, qui nous offre le plus merveilleux chef-d'oeuvre du Créateur, sans parler des différentes articulations dont la parole est formée. Que V.A. daigne seulement réfléchir sur les diverses voyelles, que la bouche prononce ou chante tout simplement. Quand on prononce ou chante la lettre $a$, le son est tout autre, que si l'on prononçoit ou chantoit la lettre $e$, ou $o$, ou $i$, ou $u$, ou $a i$, etc.: quoiqu'on demeure au même ton. Ce n'est donc pas dans la rapidité ou l'ordre des vibrations qu'on doit chercher la raison de cette différence: cette raison semble si cachée que les Philosophes ne l'ont pas encore pu approfondir.

V.A. s'appercevra aisément, que pour prononcer ces diverses voyelles, il faut donner à la cavité de la bouche une différente conformation à laquelle notre bouche est propre, préférablement à celle de tous les animaux. Aussi voyons nous, que quelques oiseaux qui apprennent à imiter la voix humaine, ne sont jamais capables de prononcer distinctement les différentes voyelles; ce n'est toujours à cet égard qu'une imitation très imparfaite.

On trouve dans plusieurs orgues un registre, qui porte le nom de voix humaine. Ordinairement ce ne sont que des sons, qui rendent la voyelle ai ou ae. Je ne doute pas qu'en y faisant quelque changement on pourroit aussi produire les sons des autres voyelles $a, e, i, o, u, o u$, mais tout cela ne suffiroit pas encore pour imiter une seule parole de la voix humaine; comment y voudroit-on ajouter les lettres consonantes, qui sont autant de modifications des voyelles? Notre bouche est si admirablement ajustée, que quelque commun que soit cet usage, il nous est presque impossible d'en approfondir le véritable méchanisme.

Nous observons bien trois organes, pour exprimer les consonantes: les levres, la langue et le palais; mais le nez y concourt aussi très essentiellement. En fermant le nez on ne sauroit prononcer les lettes $m$ et $n$, on n'entend alors que $b$ et $d$. Une grande preuve de la merveilleuse structure de notre bouche qui la rend

\footnotetext{
${ }^{60}$ Aus dem zweiten Band der Lettres à une princesse d'Allemagne ..., zitiert nach OO, III.12, S. 11-12.
} 
propre à prononcer des paroles est sans doute, que l'adresse des hommes n'a encore pu réussir à l'imiter par quelques machines. On a bien imité le chant, mais sans aucune articulation de sons et distinction des diverses voyelles.

Ce seroit sans doute une de plus importantes découvertes, que de construire une machine qui fut propre à exprimer tous les sons de nos paroles avec toutes les articulations. Si l'on réussissoit jamais à exécuter une telle machine, et qu'on fût en état de lui faire prononcer toutes les paroles par le moyen de certaines touches, comme d'une orgue ou d'un clavecin, tout le monde seroit avec raison surpris d'entendre, qu'une machine prononçât des discours entiers, ou des sermons qu'il seroit possible d'accompagner avec la meilleure grace. Les prédicateurs et les orateurs, dont la voix n'est pas assez forte ou agréable, pourroient alors jouer leurs sermons et discours sur une telle machine, tout de même que les organistes jouent des pieces de musique. La chose ne me paroit pas impossible.

4.2. Französisches Original der zitierten Stelle aus Eulers Brief an Lambert vom 18.8. $1761^{61}$ :

Vous trouverez, Monsieur, dans les Miscell. de Turin les principes de la véritable Théorie sur la propagation du mouvement par un milieu élastique, et l'application à la propagation du son. Cette matière demande pourtant encore des recherches bien subtiles; jusqu'ici on n'a considéré les sons que par rapport au grave et l'aigu mais on y remarque tant d'autres différences, par exemple deux sons, dont l'un prononce la voyelle $a$ et l'autre $e$, ou $i$, ou $o$, ou $u$ sont différens, quoiqu'ils soient également aigus ou graves; et je ne sais pas si personne s'est donné la peine de chercher, enquoi consiste la différence de ces sons et enquoi l'agitation dans l'air est différente ; ne pourroit-on pas faire des machines, qui prononceroient distinctement ces voyelles, ce qui ne manqueroit pas de produire un bon effet. Peut- être parviendroit-on enfin à les modifier par les consonnes; et à pousser la mécanique jusqu'à faire une machine qui prononceroit distinctement des paroles entières, et même des harangues, ce qui seroit excellent pour les prédicateurs, dont la voix n'est pas assez forte ou assez agréable. Dans ces recherches on n'auroit qu'à suivre la nature et l'organisme de notre langage, et cela serviroit à nous en faire connoitre mieux tous les ressorts qu'on n'a que trop negligés jusqu'ici, et qui fournissent pourtant des sujets très dignes de notre attention ; ensuite comment toutes ces différences sont elles aperçues par l'oreille? il me semble que

\footnotetext{
${ }^{61}$ [11, S. 27]. Der erste Satz des folgenden Zitats bezieht sich wohl ebenso auf die grossen Arbeiten des jungen Lagrange zur Ausbreitung des Schalls: Recherches sur la nature de la propagatoin du son, (Mélanges de philosophie et de mathématique de la société royale de Turin 1 (Oeuvres de Lagrange, ed. Serret, I, S. 39-148) als auch auf Eulers Reaktion E268: Lettre de M. Euler à M. de la Grange; Recherches sur la propogation des ébranlemens dans une [sic] milieu élastique, Mélanges de philosophie et de mathématique de la société royale de Turin 2. Lagranges Folgearbeit: Nouvelles recherches sur la nature de la propagation du son, Mélanges de philosophie et de mathématique de la société royale de Turin 2 (Oeuvres de Lagrange, ed. Serret, I, S. 151-316) wird in späteren Briefen zwischen Euler und Lambert diskutiert.
} 
dans toute cela les Physiciens ne connoissent encore presque rien. Voilà bien des questions, qui mériteroient tous les soins des Savans.

4.3. Lambert an Euler, Ende des Briefes vom 12.7.1762 ${ }^{62}$ :

Il semble que la difference des sons, cō̄e par ex. des voielles a quelquechose de specifique, et que peutêtre on pourroit mieux la comparer à la difference des couleurs et de leur melange, que les differens intervalles des sons, come on a fait jusqu'ici. L'organisme pour prononcer les voielles sans consonnes se distingue aisement. C'est principalement la langue, qui les modifie, puisqu'en prenant entre les dents un bois pour en conserver l'ouverture, ou en fermant les dents tout à fait, on peut encore les prononcer assez distinctement et à l'unisson, bienque cela aille mieux, en y emploiant les dents et le levres. On donne par cet organisme une certaine figure de l'onde, qui transmet le son, dont l'ouverture des levres et des dents semble être la base. Par contre les sons aigus ou graves dépendent plutôt de la gorge, puisque on chantera la même voielle par exemple $a$ par toutes les notes, sans mouvoir ni dents ni levres, et sans autre mouvem ${ }^{\mathrm{t}}$ de la Langue que celui qu'entraîne le retercissement successif de la Gorge. Ensuite la force d'un même son et prononcé par une même voielle peut être diférente, à mesure que le poumon et les muscles sont plus susceptible de tension, de force et de vitesse. La differente âpreté des surfaces interieurs de la gorge fait enfin la difference entre les sons plus ou moins sonores. Les flutes traversières sont sujettes à ce changement, si elles ne sont bien huilées et nettoiées interieurement. Du reste l'air, qui touche i mediatement le tympane et le canaux de l'oreille aiant plus de Chaleur que l'air exterieur, est aussi different en densité, et je ne doute pas que cela ne contribue à modifier encore les sons, jusqu'à faciliter ou à affoiblir l'ouie. L'obliquité de l'incidence des sons dans l'oreille nous fait assez demêler la contrée d'où ils viennent, et il y a apparence, que les nerfs, qui transmettent le son jusqu'au cerveau sont mus differemment par cette obliquité, et que cette différence va jusqu'à être locale.

4.4. Aus Eulers Antwort vom 4.12 .1762 auf das letztzitierte Schreiben Lamberts ${ }^{63}$ :

La vraye difference entre les sons des diverses voyelles meriteroit bien le plus soigneux examen: je crois qu'il en faut chercher la raison dans l'étendue et la figure de la masse d'air qui a été ébranlé originairement; et la manière, dont cette massse est enfermée ou ouverte y contribuera sans doute aussi beaucoup, mais que la diversité des voyelles reponde à celle des couleurs me paroit encore très incertain, ne voyant point, comment une plus grande ou plus petite

\footnotetext{
${ }^{62}[11$, S. 30]. Dies ist der direkte Antwortbrief auf das zuvor zitierte Schreiben Eulers, nach fast einem Jahr - es war freilich ein ereignisreiches Jahr, das die grosse Wende zugunsten Preussens im Siebenjährigen Krieg brachte.

${ }^{63}[11$, S. 32].
} 
refraction en pourroit être l'effet; quoique la meme diffulté se trouve egalement, quand on compare les diverses couleurs aux sons graves et aigus; si ce n'est qu'une experience attribuée à $\mathbf{M}^{\mathrm{r}}$. Demairan soit fondée, que les sons aigues se propagent plus promptement que les graves.

4.5. Im Antwortschreiben hierauf spricht sich Lambert noch einmal dafür aus, die Verschiedenheit der Vokale mit den Farben zu vergleichen ${ }^{64}$ :

Une des raisons pourquoi j'avois cru pouvoir comparer dans ma précedente les voielles aux couleurs, c'est que la différence de l'une et de l'autre semble être specifiq. On reconnoit les voielles comme on reconnoit les couleurs. Mais les degrés de clarté et ceux de l'intensité des sons ne se reconnoissent pas si aisément. Et souvent en croiant chanter telle ou telle note on se trouve trompé en consultant quelq. Instrument. J'étois sur le point de faire cette reamarque S14 de la Photometrie. Il semble que les couleurs ne sont que des modifications des mêmes raions, produites dans les corps diaphanes et colorés, et que les sons de voielles ne different point autrement.

4.6. Im „Zweyten Band“ seines Neues Organon oder Gedanken über die Erforschung und Bezeichnung des Wahren und dessen Unterscheidung von Irrthum und Schein, Leipzig (Johann Wendler) 1764 beschäftigt sich Johann Henrich Lambert mit der ,Semiotik oder Lehre von Bezeichnung der Gedanken und Dinge“ und der Bedeutung der menschlichen Sprache:

$\S 15$ (S. 12): Bey so bewandten Sachen ist nicht zu bezweifeln, daß die Natur des Menschen ganz dazu eingerichtet sein sollte. In der That umgiebt uns die Luft, welche den Schall fortpflanzet, aller Orten, und so, daß wir ohne dieselbe nicht leben könnten. Die meisten lernen von dem mündlichen Vortrage leichter, als wenn sie eben denselben lesen müßten, und viele können, ohne laut zu lesen, mit dem Lesen nicht fortkommen. Der Schall ist ferner von der Art, daß man selten genöthigt ist, das Ohr nach demselben zu wenden, wie wir das Auge gegen die Sache richten müssen: und da die Gränzen des deutlichen Sehens innerhalb wenigen Zollen enthalten sind, so können wir den Schall in ungleich größern Entfernungen vernehmlich hören, und die Rede durch merklich viele Stuffen verstärken ...

4.7. Bei der Diskussion der Feinstruktur der (mündlich-) sprachlichen Zeichen unterscheidet Lambert 17 Vokale $^{65}$ :

\footnotetext{
${ }^{64}$ Vom 7.3.1763, [11, S. 34].

${ }^{65}$ In Eulers Nachlass fand sich ebenfalls eine Arbeit, die sich mit einer genaueren Klassifizierung der Vokale in Euler bekannten Sprachen beschäftigt, zu denen das Deutsch-Schweizerische, das Schwäbische und Bayerische ebenso gehören wie Griechisch und Hebräisch: E852, Meditationes de formatione vocum (OO, III.1).
} 
$\$ 74$ (S. 47): Auf diese Weise wird es schwer, die mögliche Anzahl der einfachen Buchstaben zu bestimmen. Wir wollen bey den Vokalen anfangen, und bemerken, daß sie nur stufenweise von einander verschieden sind, und daher wegen der Continuität dieser Stufen nicht wohl auf eine bestimmte Zahl gebracht werden können, weil es ein feines Gehör erfordert, die kleinern Unterschiede zu bemerken. Die größern Unterschiede sind, so viel mir vorgekommen, folgende:

1. a, wie es die Deutschen in haben, Adam etc. aussprechen.

2. oa, ein Mittelton zwischen o und a.

3. ae, wie in dem Wort Vers, mäß, her.

4. ae, wie im Französischen in fait, im Deutschen sey, Herr.

5. e, wie in geh, mehr,

6. e, wie in den letzten Sylben der Wörter sitzen, faire. 7. e, wie in einigen holländischen Oertern die erste Sylbe in siten, ein Mittelton zwischen e und i.

8. i, wie im Deutschen mir, Hirt.

9. i, wie im Französischen vif, im Deutschen vil.

10. u, wie im Französischen pur.

11. u, wie in der Schweiz für, über, füllen.

12. u, wie outre, joug, Muse, Uhr.

13. u, wie gloire, ruhen, fuhr.

14. u, wie Stufe, murren, um.

15. o, das o chiuso der Italiener, ein Mittel zwischen u und o.

16. o, das o aperto der Italiener, ein klingendes o.

17. oe, wie inder Schweiz hören, im Französischen leur, feu.

4.8. Französisches Original der Stelle aus La Mettries L'Homme Machine ${ }^{66}$ :

Qu'on m'accorde seulement que la Matière Organisée est douée d'un principe moteur, qui seul la différentie de celle qui ne l'est pas ... \& que tout dépend dans les Animaux de la diversité de cette Organsiation, comme je l'ai assez prouvé; c'en est assez pour deviner l'Enigme des substances \& celle de l'Homme. On voit qu'il n'y a qu'une dans l'Univers \& que l'Homme est la plus parfaite. Il est au singe, aux Animaux les plus spirituels, ce que le Pendule Planétaire de Huygens, est à une Montre de Julien le Roi. S'il a fallu plus d'instrumens, plus de Rouages, plus de ressorts pour marquer les mouvements des Planètes, que pour marquer les Heures, ou les repeter; s'il a fallu plus d'art à Vaucanson pour faire son fluteur, que pour son Canard, il eût dû en emploier encore davantage pour faire une Parleur; Machine qui ne peut plus être regardée comme impossible, surtout entre les Mains d'un nouveau Prométhée.

${ }^{66}$ Nach der Ausgabe Leiden (Elie Luzac) 1748, S. 92-93. 


\section{Literatur}

[1] Ayoub, R.: Euler and the Zeta Function. Amer. Math. Monthly 81 (1974), 1067-1086.

[2] Bradley, R.E.; Sandifer, C.E. (eds.): Leonhard Euler: Life, Work and Legacy. Elsevier, Amsterdam etc. 2007.

[3] Boccadoro, B.: Automate. In: Delon, M. (dir.): Dictionnaire européen des lumières. Paris (PUF/Quadrige) 2007, 147-149.

[4] Bohlmann, G.: Übersicht über die wichtigsten Lehrbücher der Infinitesimalrechnung von Euler bis auf die heutige Zeit. Jahresber. Deutsch. Math.-Verein. 6 (1897), 91-110.

[5] Burkhardt, H.: Entwicklungen nach oscillierenden Functionen und Integration der Differentialgleichungen der mathematischen Physik. Jahresber. Deutsch. Math.-Verein. 10 (1901), 1-176.

[6] Burkhardt, J.J.; Fellmann, E.A.; Habicht, W. (Hrsg.): Leonhard Euler 1707-1783. Beiträge zu Leben und Werk. Gedenkband des Kantons Basel Stadt. Birkhäuser, Basel 1983.

[7] Cooke, R.: Henri Lebesgue and René Baire. Three books on mathematical analysis (1904-1906). In: Grattan-Guinness, I. (ed.): Landmark Writings in Western Mathematics. Elsevier, Amsterdam etc. 2005, 757-777.

[8] Edwards, H.M.: Euler's definition of the derivative. Bull. Amer. Math. Soc. 44 (2007), 575-580.

[9] Engelsman, St.B.: Families of curves and the origins of partial differentiation. North-Holland, Amsterdam etc. 1984.

[10] Leonhard Euler \& Christian Goldbach. Briefwechsel 1729-1764. Herausgegeben und eingeleitet von Juškevič, A.P; Winter, E.. Akademie-Verlag, Berlin 1965.

[11] Leonhard Euler \& Johann Heinrich Lambert. Leonhard Eulers und Johann Heinrich Lamberts Briefwechsel. Bopp, K. (Hrsg.), Abhandlungen der preußischen Akademie der Wissenschaften, Phys.-math. Klasse, Nr. 2, 1924.

[12] Ferraro, G.: Some aspects of Euler's theory of series: Inexplicable functions and the Euler-MacLaurin summation formula. Historia Math. 25 (1998), 290-317.

[13] Fraser, C.: The Calculus as Algebraic Analysis: Some observations on mathematical analysis in the $18^{\text {th }}$ century. Arch. Hist. Exact Sci. 39 (1989), 317-335.

[14] Godefroy, M.: La fonction gamma; théorie, histoire, bibliographie. Gauthier-Villars, Paris 1901.

[15] Goldenbaum, U.: Appell an das Publikum. Die öffentliche Debatte in der deutschen Aufklärung 16871796. 2 Bände. Akademie-Verlag, Berlin 2004.

[16] Goldstein, C.; Schappacher, N.: A book in search of a discipline. In: The Shaping of Arithmetic after C.F. Gauss's Disquisitiones Arithmeticae. Springer, Heidelberg etc. 2007, 3-65.

[17] Hofmann, J.E.: Eulers erste Reihenstudien. In: Schröder, K. (Hrsg.): Sammelband der zu Ehren des 250. Geburtstages Leonhard Eulers der deutschen Akademie der Wissenschaften zu Berlin vorgelegten Abhandlungen. Akademie-Verlag, Berlin 1959, 139-208.

[18] Jacobi, C.G.J.: De usu theoriae integralium ellipticorum et integralium abelianorum in analysi diophantea. Crelle J. Reine Angew. Math. 13 (1835), 353-355.

[19] Jahnke, H.N. (Hrsg.): Geschichte der Analysis. Spektrum-Verlag, Heidelberg, Berlin 1999.

[20] Kneser, A.: Das Prinzip der kleinsten Wirkung von Leibniz bis zur Gegenwart. Reihe: Wissenschaftliche Grundfragen, Philosophische Abhandlungen. Teubner, Leipzig, Berlin 1928.

[21] Liszt, F.: Correspondance. Lettres choisies, présentées et annotées par Pierre-Antoine Huré et Clauden Knepper. Jean-Claude Lattès, Paris 1987.

[22] Nick, K.R.: Kontinentale Gegenmodelle zu Newtons Gravitationstheorie. Diss., Frankfurt 2001.

[23] Petri, B.; Schappacher, N.: On Arithmetization. In: The Shaping of Arithmetic after C.F. Gauss's Disquisitiones Arithmeticae. Springer, Heidelberg etc. 2007, 343-374. 
[24] Poincaré, H.: Sur les propriétés arithmétiques des courbes algébriques. Journal de Mathématiques, $5^{\text {ème }}$ série, t. 7, fasc. III (1901), 161-233.

[25] Standage, T.: The Turk. The life and times of the famous eighteenth-century chess-playing machine. Walker \& Co., New York 2002.

[26] Schaffer, S.: Enlightened Automata. In: Clark, W.; Golinski, J.; Schaffer, S. (eds.): The Sciences in Enlightened Europe. The University of Chicago Press, Chicago \& London 1999, 126-165.

[27] Schappacher, N.: Développement de la loi de groupe sur une cubique. Séminaire de Théorie des Nombres Paris 1988/89; Progr. Math. 91 (1991), 159-184

[28] Splinter, S.: Zwischen Nützlichkeit und Nachahmung. Eine Biographie des Gelehrten Christian Gottlieb Kratzenstein (1723-1795). Peter Lang, Frankfurt a.M. 2007.

[29] Weil, A.: Number Theory. An approach through history, from Hammurapi to Legendre. Birkhäuser, Basel 1984.

[30] Wellman, K.: La Mettrie. Medicine, Philosophy, and Enlightenment. Duke University Press, Durham, NC 1992.

[31] Willis, R.: An Attempt to Analyse the Automaton Chess Player of Mr. De Kempelen. To which is added a copious collection of The Knight's Moves over the chessboard. London 1821.

\section{Norbert Schappacher}

U.F.R. de mathématique et d'informatique

I.R.M.A.

7, rue René Descartes

F-67084 Srasbourg

e-mail: schappa@math.u-strasbg.fr 